sex and baseline anthropometrics. Energy intake was significantly correlated with weight gain $(r=+0.31, p<0.001)$, length gain $(r=+0.20$, $\mathrm{p}<0.001)$ and head circumference growth $(\mathrm{r}=+0.26, \mathrm{p}<0.001)$. In addition, protein intake was independently positively associated with weight gain, and the effect of macronutrient intakes on growth varied with postnatal age.

Conclusions Extremely preterm infants in Sweden received lower macronutrient intakes than recommended and showed postnatal growth failure. We found significant positive correlations between intakes of energy and macronutrients and growth. This suggests that optimized energy and macronutrient intake is important to prevent growth failure in these vulnerable infants.

\section{EFFECTS OF DIFFERENT FATTY ACIDS ON RED BLOOD CELL MORPHOLOGY}

doi:10.1136/archdischild-2012-302724.0350

'J Derganc, 'V Arrigler, 'M Marolt, 'M Mlinaric, ${ }^{2} \mathrm{M}$ Derganc. ${ }^{1}$ Institute of Biophysics, University of Ljubljana Medical Faculty; ${ }^{2}$ Dept. of Pediatric Surgery and Intensive Care, University Medical Centre Ljubljana, Ljubljana, Slovenia

Background and Aims Recently echinocytosis and subsequent haemolytic anaemia was described in a premature infant receiving omega-3 fatty acids (Omegaven) in parenteral nutrition. It was presumed that omega-3 fatty acids caused echinocytosis. No study has been done to compare the effect of different fatty acids used in parenteral nutrition on human red blood cell (RBC) morphology. We therefore studied the effect of omega- 3 fatty acids (Omegaven) and omega -6 fatty acids (Intralipid) at different concentrations on RBC in vitro.

Methods Blood samples were obtained from 12 healthy adult volunteers. Aliquots with $0.5 \mathrm{ml}$ of washed RBC resuspended in autologous plasma to a hematocrit of $48 \%$ and containing $0 \%, 5 \%, 10 \%$, $20 \%, 30 \%$ and $40 \%$ of Omegaven or Intralipid were prepared and incubated for $30 \mathrm{~min}$ at $37^{\circ} \mathrm{C}$. The cells were then fixed with $1 \%$ glutaraldehyde and inspected under an inverted brightfield microscope. The extent of echinocytosis was quantified by means of the morphological index (MI), calculated according to the standard protocol.

Results It was found that at concentrations equal to and higher than $20 \%$, Omegaven produced significantly higher RBC morphological index (MI) than Intralipid: mean MI at $20 \%$ for Intralipid was $0.61 \pm 0.24$ and for Omegaven $1.12 \pm 0.43$ ( $p<0.01$ ), whereas at $40 \% \mathrm{MI}$ was $1.47 \pm 0.37$ and $2.48 \pm 0.66$ for Intralipid and Omegaven, respectively $(\mathrm{p}<0.01)$.

Conclusions At concentrations over 20\% Omegaven is more likely to cause echinocytosis than Intralipid. The higher concentrations may occur in vivo if Omegaven is given separately from other parenteral nutrition fluids (two-in one).

\section{DIFFERENTIAL REGULATION OF CORD BLOOD NESFATIN-1 IN LARGE FOR GESTATIONAL AGE PREGNANCIES}

doi:10.1136/archdischild-2012-302724.0351

'DD Briana, 'G Kafalidis, 'T Boutsikou, 'M Boutsikou, ${ }^{2} \mathrm{D}$ Piatopoulou, 'S Baka, 'D Hassiakos, ${ }^{2} \mathrm{D}$ Gourgiotis, 'A Malamitsi-Puchner. 'Neonatal Division; 2nd Department of Obstetrics and Gynecology; ${ }^{2}$ Research Laboratory of Clinical Biochemistry-Molecular Diagnostics; 2nd Department of Pediatrics, Athens University Medical School, Athens, Greece

Background and Aims Large-for-gestational-age (LGA) infants are at increased risk for developing alterations in metabolic programming, which may lead to impaired glucose homeostasis during infancy, childhood and adulthood. Adipocytokines play an important role in short- and long-term energy balance, insulin resistance and fetal growth. The objective of the present study was to investigate circulating concentrations of nesfatin-1 (novel adipocytokine, also expressed by the gastric mucosa and pancreatic $\beta$-cells) in fetal samples from LGA and appropriate-for-gestational-age-(AGA) pregnancies and study their association with gender, parity, and delivery mode.

Methods Cord blood nesfatin-1 concentrations were prospectively measured by enzyme-linked immunosorbent assay in 40 LGA ( 9 born from diabetic mothers and 31 born from non-diabetic mothers) and 20 AGA singleton full-term infants.

Results Cord blood nesfatin-1 concentrations were lower in LGA compared to AGA neonates, after controlling for confounding factors $(b=-0.206, p=0.005$, SE 0.07$)$. However, cord blood nesfatin- 1 concentrations were elevated in infants born from mothers presenting with gestational diabetes mellitus (GDM), compared to those born from non-diabetic mothers, after controlling for group $(b=0.190, p=0.050, S E 0.10)$. Finally, cord blood nesfatin-1 concentrations were lower in cases of vaginal delivery $(b=0.11, p=0.042$, SE 0.05).

Conclusions Down-regulation of nesfatin-1 in LGA fetuses probably represents a negative feedback exerted by adipose tissue on nesfatin-1 production. On the other hand, fetal nesfatin-1 concentrations are higher in cases of GDM, probably indicating the possible involvement of nesfatin-1 in the regulation of insulin secretion from pancreatic $\beta$-cells. Finally, vaginal delivery-associated inflammation could probably account for lower cord blood nesfatin-1 concentrations.

\section{LONGITUDINAL INFUSION OF INSULIN-LIKE GROWTH FACTOR-I AND IGF-BINDING PROTEIN-3 COMPLEX TO FIVE PRETERM INFANTS - PHARMACOKINETICS AND SHORT TERM SAFETY}

doi:10.1136/archdischild-2012-302724.0352

${ }^{1} \mathrm{D}$ Ley, $1 \mathrm{I}$ HansenPupp, ${ }^{2} \mathrm{~A}$ Niklasson, ${ }^{3} \mathrm{M}$ Domellöf, ${ }^{4} \mathrm{~L}$ Friberg, ${ }^{5} \mathrm{~J}$ Borg, ${ }^{2} \mathrm{C}$ Löfquist, ${ }^{2} \mathrm{G}$ Hellgren, ${ }^{6} \mathrm{~L}$ Smith, ${ }^{2} \mathrm{AL}$ Hård, ${ }^{2} \mathrm{~A}$ Hellström. 'Lund University, Lund; ${ }^{2}$ Gothenburg University, Gothenburg; ${ }^{3} U$ meå University, Umeå; ${ }^{4}$ Uppsala University; ${ }^{5}$ Premacure $A B$, Uppsala, Sweden; ${ }^{6}$ Harvard Medical School, Boston, MA, USA

Introduction In preterm infants, low levels of insulin like growth factor-I (IGF-I) and IGF binding protein 3 (IGFBP-3) are associated with impaired brain growth and retinopathy of prematurity (ROP).

Treatment with IGF-I/IGFBP-3 may be beneficial for brain development and decrease the prevalence of ROP.

Methods In a phase II pharmacokinetic and safety study, five infants ( 3 girls) with a median (range) gestational age (GA) of $26+6$ $(26+0-27+2)$ weeks and birth weight (BW) of 990 (900-1212) g received continuous intravenous infusion of rhIGF-I/rhIGFBP-3. Treatment was initiated during the first postnatal day and continued for a median (range) duration of $168 \mathrm{~h}$ (47-168) in doses between $21-111 \mu \mathrm{g} / \mathrm{kg} / 24 \mathrm{~h}$.

Results Treatment with rhIGF-I/rhIGFBP-3 was associated with higher serum IGF-I and IGFBP-3 concentrations $(p<0.001)$ than model-predicted endogenous levels. Out of 74 IGF-I samples measured during study drug infusion, $37(50 \%)$ were within target range, $4(5 \%)$ above and $33(45 \%)$ were below. Predicted dose of rhIGF-I/rhIGFBP-3 to establish circulating levels of IGF-I within the intrauterine range in a $1000 \mathrm{~g}$ infant was $75-100 \mu \mathrm{g} / \mathrm{kg} / 24 \mathrm{~h}$. No hypoglycemia or other adverse effects were recorded.

Discussion Continuous intravenous infusion of rhIGF-I/ rhIGFBP-3 was effective in increasing serum concentrations of IGF-I and IGFBP-3. Administration under study conditions was safe.

353 PRETERM BIRTH AND THE METABOLIC SYNDROME: A SYSTEMATIC REVIEW AND META-ANALYSIS

doi:10.1136/archdischild-2012-302724.0353 